Background Non-communicable diseases are increasingly recognised as the major health issue facing many governments. Brazil has observed a rapid change in the weight profiles of its population, with the prevalence of obesity and overweight increasing partly as a result of the well documented effect of the nutrition transition. Obesity-related diseases are placing a substantial health and economic burden on the country. While the problem has been recognized, the implications of current trends on future overweight and obesity rates, BMI-related disease and costs associated with that disease burden have not been considered.

Methods A previously developed micro-simulation model was used to project through the year 2050 the extent of obesity, BMI related diseases, and associated health care costs in Brazil. A MonteCarlo simulation method has been utilized to simulated BMI related diseases. In total, thirteen conditions were modelled: coronary heart disease, stroke, hypertension, diabetes, knee osteoarthritis, and eight cancers (breast, kidney, colorectal, oesophageal, endometrial, gallbladder, liver and pancreas). The authors also projected a possible decrease in the BMI and its impact on health and health care costs.

Results In 2010, nearly 45\% of the Brazilian male population were overweight or obese (BMI $\geq 25 \mathrm{~kg} / \mathrm{m}^{2}$ ), but by 2050 we project rates as high as $95 \%$. A slightly less pessimistic picture is observed among females: $42 \%$ in 2010 increasing to $52 \%$ in 2050 . The disease incidence figures increase considerably due to obesity patterns. However, BMI reduction across the population will alter these disease projections. Nearly three million diabetes cases and USD 388 million in health care expenditure can be avoided with a five percent reduction in BMI alone by 2050.

Conclusion Obesity rates are rapidly increasing in Brazil creating a high burden of diseases and associated health care costs. However, even a one and percent reduction in prevalence rates will substantially reduce the disease and cost burden. Though some steps have been taken for tackling the obesity problem, Brazil still needs a strong, comprehensive policy involving multiple agencies and institutions with strong leadership.

\section{OP22 PROJECTIONS OF HEALTHCARE COSTS IN THE LAST YEAR OF LIFE FOR OLDER CANCER PATIENTS IN EUROPEAN UNION MEMBER STATES (2008-2030)}

doi:10.1136/jech-2012-201753.022

H Petkova, D Dawoud, P McCrone, IJ Higginson. 'Department of Palliative Care, Policy and Rehabilitation, King's College London, London, UK; '2Department of Palliative Care, Policy and Rehabilitation, King's College London, London, UK; ${ }^{3}$ Health Service and Population Research Department, King's College London, Institute of Psychiatry, London, UK; ${ }^{4}$ Department of Palliative Care, Policy and Rehabilitation, King's College London, London, UK

Background Despite advances, cancer remains a major cause of pain, suffering and death. The highest incidence and mortality occurs in older people $(\geq 65)$, and given demographic change the numbers are expected to rise in the coming years. This has financial consequences and key implications for service planning. We estimated the current and future cost of providing care for older cancer patients in the last year of life (LYOL) in the 27 European Union (EU) member-states between 2008 and 2030.

Methods Our cost projection models combined: a) WHOGLOBOCAN mortality data; b) an average UK-based estimate of the cost of care per cancer patient in the LYOL; c) country-specific adjustment factor; d) annual cost inflation correction range 5\%-15\%: 5\% (conservative scenario), 10\% (realistic scenario), 15\% (highest expected increase scenario), and e) an adjustment for ageing to reflect the increasing life expectancy (LE) and the related morbidity expansion.

Results The total EU cost of care for older cancer patients in the LYOL is expected to increase by 48\% (2008-2030) reflecting mortality rates only (baseline model). Assuming 5\% cost rise per year (model 1), we projected more than a two-fold increase in the total EU cost by 2030 at an average annual rate of $9.6 \%$. Model 2 involved $10 \%$ inflation and no ageing-adjustment, predicting a 3.7 times cost rise during the next two decades. This equated an annual average increase at $16.9 \%$. We predicted a slightly higher cost rise when we allowed for ageing in addition to the $10 \%$ annual inflation (model 3) at approximately $17 \%$ increase year on year. This represents $\$ 81$ billion absolute increase during the projected period or $\$ 3.6$ average annual increase for Europe as a whole. The highest cost increase is expected in model 4 (15\% inflation with ageing-adjustment). Costs are predicted to rise more than five times between 2008 and 2030, marking a $24.3 \%$ average annual increase. There is a marked cost variation across Europe.

Conclusion Costs of care for older cancer patients in the LYOL are projected to rise substantially in the foreseeable future. Our models outline cost variation between $2.2 \%$ and $24.3 \%$, with the most realistic increase expected at $17 \%$ annually. This has key implications for short and medium-term service planning of how to provide care in the LYOL for the rising number of older citizens dying from cancer, taking into account the current economic climate in Europe.

\section{OP23 DEVELOPING A SINGLE, EVIDENCE-BASED APPROACH TOWARDS DISINVESTMENT: IS A NICE WAY THE RIGHT WAY?}

doi:10.1136/jech-2012-201753.023

H Flynn, CA Gericke. PenCLAHRC, The Universities of Plymouth and Exeter, Plymouth, UK

Background With the NHS currently facing one of its toughest periods financially, most, if not all, PCTs in England have started to prioritise services and sought to identify those which are either ineffective or of low clinical value for disinvestment. Yet the absence of a nationally accepted list of identified procedures has resulted in PCTs developing their own approaches, with many applying different priority thresholds to different procedures. This has created numerous "black lists" of interventions which will either not be funded or their commissioning severely curtailed. As these decisions are being based on the affordability of procedures rather than clinical efficacy, a post-code lottery in provision has ensued across the UK. Therefore to ensure equitable access to healthcare resources, an evidence based approach towards disinvestment is imperative.

Methods Working in collaboration with NICE R\&D, the project used NICE's 'do not do' (DND) database to identify topics based on rigorous evidence for potential disinvestment. DNDs based upon those treatments with evidence suggesting they are ineffective or of low-clinical value were identified from the $850+$ DND database. DNDs relating solely to clinical safety were excluded from the study. Procedures were further prioritised by 34 clinical experts in participating 'pilot' implementation sites in the SW Peninsula, who were asked to review populated lists of DNDs to ensure: clinical soundness; practicality of implementation; and any DND of potential high impact locally. Using HES data, volumes of DNDs still being performed in the NHS were also collated to give an estimate of overall usage and potential impact. Each DND identified was subjected to a cost analysis to provide an estimate of potential savings at both a local and national level.

Results 209 DND topics developed between 2007 and 2012 were identified for use in the study. Following local clinical prioritisation, cost analysis and exploration of HES data to give an estimate of usage, 30 procedures for a pilot disinvestment programme were identified solely from the NICE DND database.

Conclusion The study successfully developed a single, evidencebased approach towards disinvestment through the identification of ineffective and low clinical value treatments from the NICE DND database. Additional planned research which will implement the 'pilot' list across the SW Peninsula, will provide valuable information 